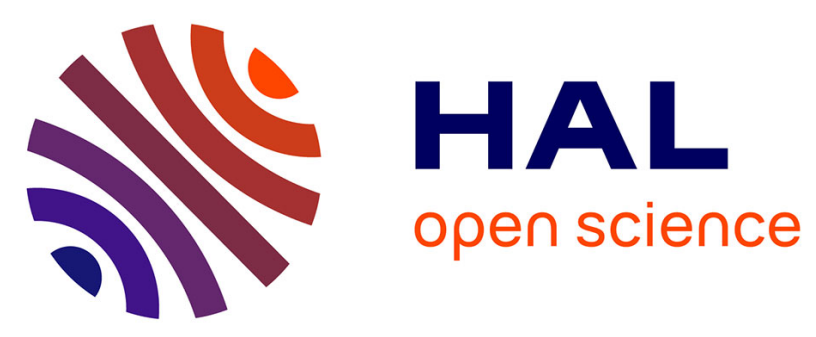

\title{
Performance of the Low-Rank Adaptive Normalized Matched Filter Test Under a Large Dimension Regime
}

Alice Combernoux, Frédéric Pascal, Guillaume Ginolhac

\section{To cite this version:}

Alice Combernoux, Frédéric Pascal, Guillaume Ginolhac. Performance of the Low-Rank Adaptive Normalized Matched Filter Test Under a Large Dimension Regime. IEEE Transactions on Aerospace and Electronic Systems, 2019, 55 (1), pp.459-468. 10.1109/TAES.2018.2847911 . hal-01825231

\section{HAL Id: hal-01825231 \\ https://hal.science/hal-01825231}

Submitted on 26 Feb 2020

HAL is a multi-disciplinary open access archive for the deposit and dissemination of scientific research documents, whether they are published or not. The documents may come from teaching and research institutions in France or abroad, or from public or private research centers.
L'archive ouverte pluridisciplinaire HAL, est destinée au dépôt et à la diffusion de documents scientifiques de niveau recherche, publiés ou non, émanant des établissements d'enseignement et de recherche français ou étrangers, des laboratoires publics ou privés. 
See discussions, stats, and author profiles for this publication at: https://www.researchgate.net/publication/325800122

\section{Performance of the Low-Rank Adaptive Normalized Matched Filter Test Under a Large Dimension Regime}

Article in IEEE Transactions on Aerospace and Electronic Systems · February 2019

DOI: 10.1109/TAES.2018.2847911

CITATION

1

3 authors, including:

Alice Combernoux

École Supérieure d'Electricité

11 PUBLICATIONS 26 CITATIONS

SEE PROFILE

Some of the authors of this publication are also working on these related projects:

ANR MARGARITA View project

Project G4M - Geodetection Multi Materials Multi Profession View project
READS

28

Guillaume Ginolhac

Université Savoie Mont Blanc

117 PUBLICATIONS 541 CITATIONS

SEE PROFILE 


\title{
Performance of the Low-Rank Adaptive Normalized Matched Filter Test Under a Large Dimension Regime
}

\author{
Alice Combernoux, Frédéric Pascal, Senior Member, IEEE, Guillaume Ginolhac, Member, IEEE
}

\begin{abstract}
When a possible target is embedded in a Low Rank (LR) Gaussian clutter (which is contained in a low dimensional subspace) plus a white Gaussian noise, the detection process can be performed by applying the Low-Rank Adaptive Normalized Matched Filter (LR-ANMF) which is a function of the estimated projector. In a recent work, we derived an approximate distribution of the LR-ANMF under the $\mathcal{H}_{0}$ hypothesis by using a restrictive hypothesis (the target has to be orthogonal to the clutter subspace). In this paper, we propose to determine new approximations of the Pfa and the Pd of the LR-ANMF by relaxing this restrictive hypothesis. This new derivation is based on results concerning the convergence in a large dimension regime of quadratic forms. Simulations validate our result, in particular when the tested signal is close to the clutter subspace.
\end{abstract}

Index Terms-Adaptive detection, Low-Rank Covariance Matrix, Approximate Detector Distribution, Random Matrix Theory, RADAR

\section{INTRODUCTION}

In order to detect a known signal coming from a target embedded in a Gaussian noise characterized by its unknown covariance matrix, the Generalized Likelihood Ratio Test (GLRT) is derived which leads to the well-known Kelly's detector [1]. This detector allows to choose between two hypothesis $\mathcal{H}_{1}$ (target present) and $\mathcal{H}_{0}$ (target absent). The theoretical Probability of False Alarm (Pfa) and Probability of Detection $(\mathrm{Pd})$ are derived in order to set a detection threshold for a chosen Pfa. Another approach is to derive the GLRT by assuming to know the covariance matrix and by replacing it in the detector by the Maximum Likelihood Estimator (MLE) of the theoretical covariance matrix [2] (in Gaussian, the MLE is the Sample Covariance Matrix - SCM). The theoretical performance of this detector are also provided even for subspace detectors [3], [4].

Unfortunately, an accurate estimation of the covariance matrix needs a large number of data (denoted secondary data and assumed to be iid and free signal) $K \approx 2 m$ (where $m$ is the data size) [5], [6]. To reduce this number $K$, it is possible to regularize the SCM [7] when any information on the system is available. In array processing applications, the received antenna is often symmetric which allows to take the persymmetry property of the data into account in order to divide by 2 the number of secondary data needed [8]-[10].

Alice Combernoux was with SONDRA - CentraleSupelec, email: alicecombernoux@gmail.com, Frédéric Pascal is with LSS - CentraleSupelec, email: frederic.pascal@ centralesupelec.fr, Guillaume Ginolhac is with LISTIC - Université Savoie Mont Blanc, email: guillaume.ginolhac@univ-smb.fr.
It is also possible to handle with symmetry properties of the disturbance [11], [12] to reduce this number of secondary data. In the case where the disturbance can be decomposed by a sum of two contributions, a White Gaussian Noise (WGN) and a Low-Rank (LR) Gaussian contribution (called clutter in RADAR or SONAR), the LR Adaptive Normalized Matched Filter (LR-ANMF) has been proposed [10], [13]. In this detector, the estimation of the covariance matrix is replaced by the estimation of the projector onto the clutter subspace which is obtained by the Eigenvalue Decomposition (EVD) of the SCM. When the LR subspace $r$ is small compared to the data size $m$, the numerator of the LR-ANMF has interesting properties: only $K=2 r$ secondary data are needed to obtain equivalent performance compared to the numerator of the ANMF built with the SCM and $K=2 m$ [14], [15]. This theoretical result is difficult to obtain with the LRANMF or even for other LR adaptive detectors [16]-[18]. When the LR-ANMF is built with the theoretical projector, its theoretical performance are available in [13]. But to our knowledge, the theoretical performance of the LR-ANMF at fixed dimension does not exist in the literature. Therefore, we have to resort to asymptotic methods to approximate its performance. In [19], we have proposed a result approximating the LR-ANMF distribution under the $\mathcal{H}_{0}$ hypothesis. To derive this result, several hypotheses have been used. The two first are mostly common: the norm of the steering vector $\mathbf{d}$ is assumed to be equal to 1 and the Clutter to Noise Ratio (CNR) is assumed to be large. This last assumption is realistic in many applications such as Spatio-Temporal Adaptive Processing (STAP) Radar. For the mathematical derivations, a last hypothesis is needed: the projection of the steering vector onto the theoretical interference subspace is negligible which means that the tested steering vector is not fully embedded in the clutter subspace. This hypothesis is often needed when we derive theoretical performance with a projector [10], [14], [15], [20], [21]. The result derived in [19] is interesting because it first shows the CFAR behavior of the LR-ANMF for a tested vector far from the clutter subspace and allows to quickly evaluate an approximate Pfa for a chosen threshold. But in general, the LR-ANMF is more useful when the tested steering vectors are close to the clutter subspace (such as STAP to detect slow moving targets): it will be more suitable to derive its theoretical performance in these particular conditions. We propose in this paper a new approximation of the LR-ANMF distribution under $\mathcal{H}_{0}$ and $\mathcal{H}_{1}$ (and not only $\mathcal{H}_{0}$ as in [19]) by relaxing the last hypothesis about the orthogonality between 
the steering vector and the theoretical interference subspace. The approximations for Pfa and Pd for the LR-ANMF are also deduced from this approximate distribution.

This new derivation is first based on Random Matrix Theory (RMT) results. The LR-ANMF is a function of three Quadratic Forms (QFs), $\mathbf{s}_{1}^{H} \hat{\boldsymbol{\Pi}}_{\mathrm{c}}^{\perp} \mathbf{s}_{2}$ (where $\hat{\boldsymbol{\Pi}}_{\mathrm{c}}^{\perp}$ is a projector estimate and $\mathbf{s}_{1}$ and $\mathbf{s}_{2}$ are two vectors which will be explicited later) which have been broadly studied since ten years in the signal processing community [22]-[25]. The principle is to examine the spectral behavior of an estimate of the theoretical covariance matrix, like the SCM, by RMT for obtaining its convergence, performance and asymptotic distribution in the desired convergence regime. The spectral behavior is generally studied under two different regimes: when $K$ tends to infinity, for fixed $m$ and when both the data size $m$ and $K$ tend to infinity at the same rate (denoted as large dimensional regime), i.e. $\left.\left.c_{m} \triangleq m / K \rightarrow c \in\right] 0,+\infty\right)$, for different models of the observed data as in [22], [23], [26], [25] and [24]. These results show that the QFs of the form of $\mathbf{s}_{1}^{H} \hat{\mathbf{M}}_{\mathrm{c}}^{\perp} \mathbf{s}_{2}$ do not converge to the theoretical one in the large dimensional regime. The authors of [26]-[28] have proposed new MUSIC algorithms which ensure the consistency property in this large dimensional regime. In these new algorithms, the estimated projector is replaced by a new one often called pseudoprojector in the literature.

By using these convergence results, we have first proposed in [29] to integrate the new QFs of [26]-[28] in the classical detection framework and we have shown by Monte-Carlo simulations that this new detector leads to better performance in a STAP context in particular when the target is close to the clutter. In [30], we derived a first approximation of the LR-ANMF under the $\mathcal{H}_{0}$ hypothesis but the result suffers of a main drawback: the obtained approximate distribution is not a known distribution and is a function of several random variables. Therefore Monte Carlo simulations are still needed to obtain the final result and it is impossible to have a Pfathreshold relation which easily sets the threshold for a chosen Pfa. In this paper, we propose a new result for the approximation of the LR-ANMF distribution which is related to a known distribution and allows to improve the result of [30]. Moreover, this new derivation allows to have an approximation of the LR-ANMF distribution under the $\mathcal{H}_{1}$ hypothesis. This new derivation is based on convergence results in a large dimension regime and the approximate distribution of a ratio of two random QFs proposed in [31]. We validate hereafter in this paper our theoretical result by comparing it with MonteCarlo simulations in a jamming application which consists in detecting a signal among different strong interferences. These interferences are contained in a LR subspace and therefore this application is well adapted to validate our result. But, our result will remain valid in other applications such as STAP or STAP-MIMO where the LR subspace is composed by different contributions of the ground.

Paper is organized as follows: section II presents the problem statement, section III contains the main result of the paper which is the approximate distribution of the LR-ANMF and section IV shows the results of numerical simulations.

The following convention is adopted: italic indicates a scalar quantity, lower case boldface indicates a vector quantity and upper case boldface a matrix. ${ }^{T}$ denotes the transpose operator and ${ }^{H}$ the transpose conjugate. $E[]$ is the expected value operator. $\mathcal{C N}(\mathbf{a}, \mathbf{M})$ (resp. $\mathcal{N}(\mathbf{a}, \mathbf{M})$ ) is a complex (resp. real) Gaussian vector of mean $\mathbf{a}$ and of covariance matrix $\mathbf{M}$. $\mathbf{I}_{m}$ is the $m \times m$-identity matrix. $\chi^{2}(n)$ is a Chi-square random variable with $n$ degrees of freedom. $\mathscr{N}_{c}\left(a, \sigma^{2}\right)$ is complex Gaussian random variable of mean $a$ and variance $\sigma^{2} . \sim$ means "distributed as". $\Re(a)$ and $\Im(a)$ designs the real and imaginary part of a complex number $a$.

\section{Problem Statement}

\section{A. Derivation of the $L R-A N M F$}

The stated problem is to infer if the received signal $\mathbf{x} \in$ $\mathbb{C}^{m \times 1}$, corrupted by an additive disturbance, also contains a complex signal $\alpha \mathbf{d}(\boldsymbol{\Theta})$. One also has a set of $K$ secondary data $\left\{\mathbf{x}_{k}\right\}$ which are signal free realizations of the disturbance. The two hypotheses are then:

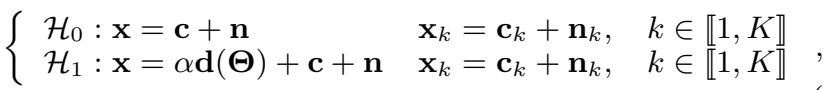

where $\mathbf{d}$ is the steering vector, $\alpha$ is an unknown deterministic parameter and $\Theta$ is an unknown deterministic vector (in the following, $\Theta$ will be omitted). $\mathbf{n} \in \mathbb{C}^{m \times 1}\left(\right.$ or $\left.\mathbf{n}_{k}\right) \sim$ $\mathcal{C N}\left(0, \gamma \mathbf{I}_{m}\right)$ is the additive WGN complex vector. $\mathbf{c} \in \mathbb{C}^{m \times 1}$ (or $\left.\mathbf{c}_{k}\right) \sim \mathcal{C N}(0, \mathbf{C})$ is the Gaussian clutter. Consequently, the covariance matrix of the secondary data can be written as $\mathbf{R}=\mathbf{C}+\gamma \mathbf{I}_{m} \in \mathbb{C}^{m \times m}$. Moreover, the clutter is considered low-rank $r^{1}$. Hence, $\operatorname{rank}(\mathbf{C})=r \ll m$ and one could write the eigendecomposition of $\mathbf{C}$ :

$$
\mathbf{C}=\sum_{i=1}^{r} \gamma_{i} \mathbf{u}_{i} \mathbf{u}_{i}^{H}
$$

where $\gamma_{i}$ and $\mathbf{u}_{i}, i \in \llbracket 1 ; r \rrbracket$ are respectively the non zero eigenvalues and the associated eigenvectors of $\mathbf{C}$. We define the projector onto the clutter subspace $\boldsymbol{\Pi}_{c}$ and the projector onto the orthogonal of the clutter subspace $\boldsymbol{\Pi}_{c}^{\perp}=\mathbf{I}_{m}-\boldsymbol{\Pi}_{c}[14]$, [15]:

$$
\begin{aligned}
& \boldsymbol{\Pi}_{c}=\sum_{i=1}^{r} \mathbf{u}_{i} \mathbf{u}_{i}^{H} \\
& \boldsymbol{\Pi}_{c}^{\perp}=\mathbf{I}_{m}-\sum_{i=1}^{r} \mathbf{u}_{i} \mathbf{u}_{i}^{H} .
\end{aligned}
$$

Since $\Pi_{\mathrm{c}}^{\perp}$ is not known in practice, we have to estimate it from the secondary data $\left\{\mathbf{x}_{k}\right\}$. The classical estimation is based on the EVD of the SCM:

$$
\hat{\mathbf{R}}=\frac{1}{K} \sum_{k=1}^{K} \mathbf{x}_{k} \mathbf{x}_{k}^{H}=\sum_{i=1}^{r} \hat{\lambda}_{i} \hat{\mathbf{u}}_{i} \hat{\mathbf{u}}_{i}^{H}+\sum_{i=r+1}^{m} \hat{\lambda}_{i} \hat{\mathbf{u}}_{i} \hat{\mathbf{u}}_{i}^{H},
$$

where $\hat{\lambda}_{i}$ and $\hat{\mathbf{u}}_{i}$ are the estimated eigenvalues and eigenvectors. Finally, the estimated projectors are:

$$
\begin{aligned}
\hat{\boldsymbol{\Pi}}_{\mathrm{c}} & =\sum_{i=1}^{r} \hat{\mathbf{u}}_{i} \hat{\mathbf{u}}_{i}^{H} \\
\hat{\boldsymbol{\Pi}}_{\mathrm{c}}^{\perp} & =\mathbf{I}_{m}-\sum_{i=1}^{r} \hat{\mathbf{u}}_{i} \hat{\mathbf{u}}_{i}^{H} .
\end{aligned}
$$

\footnotetext{
${ }^{1}$ The rank is assumed to be known in this paper such as STAP applications according to Brennan's formula [32], [33] $r=N+(M-1) * \beta(N$ is the number of sensors, $M$ the number of pulses and $\beta$ depends on radar parameters). If not, it is possible to estimate it for example with new methods based on Random Matrix Theory tools [34].
} 
The conventional adaptive test to the problem of detection (1) has been proposed in [13] and is called the LR-Adaptive NMF (LR-ANMF):

$$
\hat{\Lambda}_{\mathrm{LR}}=\frac{\left|\mathbf{d}^{H} \hat{\boldsymbol{\Pi}}_{\mathrm{c}}^{\perp} \mathbf{x}\right|^{2}}{\left(\mathbf{d}^{H} \hat{\boldsymbol{\Pi}}_{\mathrm{c}}^{\perp} \mathbf{d}\right)\left(\mathbf{x}^{H} \hat{\boldsymbol{\Pi}}_{\mathrm{c}}^{\perp} \mathbf{x}\right)} \underset{\mathcal{H}_{0}}{\stackrel{\mathcal{H}_{1}}{\gtrless}} \eta
$$

where $\underset{\mathcal{H}_{0}}{\stackrel{\mathcal{H}_{1}}{\gtrless}} \eta$ means that the $\mathcal{H}_{1}$ hypothesis (respectively $\mathcal{H}_{0}$ ) is decided if the test $\hat{\Lambda}_{\mathrm{LR}}$ is over (respectively under) the threshold $\eta$.

\section{B. Previous result on theoretical LR-ANMF distribution}

In [19], we have obtained the following result for an approximate distribution under the $\mathcal{H}_{0}$ hypothesis of $\hat{\Lambda}_{\mathrm{LR}}$.

Proposition II.1 The LR-ANMF of Eq. (6), $\hat{\Lambda}_{\mathrm{LR}}$, can be expressed as a function of 6 independent random variables:

$$
\hat{\Lambda}_{L R} \sim \hat{\Lambda}_{L R_{t h}}=\frac{|\alpha|^{2}}{\beta},
$$

with

$$
\begin{aligned}
& \alpha=b_{1}-\frac{1}{K} X_{1} X_{3} s_{1} \\
& \beta=\left|b_{1}\right|^{2}+X_{2}^{2}-\frac{2}{K} X_{1} X_{3} \Re\left(b_{1} s_{1}+X_{2} s_{2}\right)
\end{aligned},
$$

where $X_{1} \sim \sqrt{\frac{1}{2} \chi^{2}(2 r)}, X_{2} \sim \sqrt{\frac{1}{2} \chi^{2}(2(m-r-1))}, X_{3} \sim$ $\sqrt{\frac{1}{2} \chi^{2}(2 K)}, s_{1} \sim \mathcal{N}_{c}(0,1), s_{2} \sim \mathcal{N}_{c}(0,1)$ and $b_{1} \sim \mathcal{N}_{c}(0,1)$ are independent random variables.

To derive this result, several assumptions have been used. First, we assumed that the norm of steering vector $\mathbf{d}$ is equal to 1 and that the CNR is large which leads to: $\gamma_{1}, \ldots, \gamma_{r} \gg$ $\gamma$. This assumption is realistic in many applications such as STAP. The last assumption is the most restrictive but it is needed for the mathematical derivations: the projection of the steering vector onto the theoretical interference subspace is negligible, i.e. $\mathbf{u}_{i}^{H} \mathbf{d} \approx 0$ for $i=1, \ldots, r$. This means that the tested steering vector is not fully embedded in the clutter ridge. This assumption is often needed when we derive theoretical performance with projector [10], [14], [15], [20], [21].

The result of Prop. II.1 shows that the LR-ANMF is Constant False Alarm Rate (CFAR) when the tested target is far from the LR subspace. But in practice and by simulation, it is well known that this property is not guaranteed when the target is close to the LR subspace. Actually, the Pfa then depends on the distance between the tested vector and the clutter subspace. Therefore, we propose in this paper a new approximation of the LR-ANMF distribution by relaxing the last assumption about the orthogonality between the steering vector and the theoretical interference subspace which allows to obtain a valid result for any tested target. Moreover, this new derivation will allow us to obtain an approximate distribution (and not a function of several random variables) under $\mathcal{H}_{0}$ and $\mathcal{H}_{1}$ hypothesis leading to obtain the approximations for $\mathrm{Pfa}$ and Pd. The approach used in this paper is based on RMT tools and in particular on the convergences of the three QFs of Eq. (6).

\section{RMT model and convergence results}

In this subsection, we present the standard convergence results in a large dimension regime of the QFs.

1) Covariance matrix models: These convergence results are based on the behavior of the eigenvalues of the SCM when $m, K \rightarrow \infty$ at the same rate, i.e. $\left.\left.c_{m} \triangleq m / K \rightarrow c \in\right] 0,+\infty\right)$. The general assumptions that are useful to the different convergences will be first presented. Next, the covariance model used in this paper, the $G$-MUSIC model [22] [23], will be provided. The studied data models are characterized by their formulation and in a more important way by the asymptotic behavior of the covariance matrix eigenvalues when $m, K \rightarrow \infty$ at the same rate. Then, to prove convergences, we make the following standard assumptions:

(As1) $\mathbf{R}$ has uniformly bounded spectral norm $\forall m \in \mathbb{N}^{*}$, i.e. $\forall i \in \llbracket 1, m \rrbracket,\left|\lambda_{i}\right|<\infty$. More precisely, let $\mathbf{R}_{m}$ be the covariance matrix indexed by its size $m$ and $\lambda_{1, m}, \cdots, \lambda_{m, m}$ its eigenvalues. Then, we assume that $\sup _{m} \max _{1 \leqslant i \leqslant m}\left|\lambda_{i, m}\right|<\infty$.

(As2) The vectors $\boldsymbol{s}_{1}, \boldsymbol{s}_{2} \in \mathbb{C}^{m \times 1}$ used in the QFs (here $\boldsymbol{d}(\Theta)$ or $\left.\mathbf{x} /\|\mathbf{x}\|_{2}\right)$ have uniformly bounded Euclidean norm $\forall m \in \mathbb{N}^{*}$.

(As3) The elements of the Random Matrix have to be Gaus$\operatorname{sian}^{2}$.

Under the previous assumptions, the behavior of the SCM eigenvalues can be studied in a large dimension regime. By building the matrix $\mathbf{X}=\left[\mathbf{x}_{1}, \cdots, \mathbf{x}_{K}\right]$, the SCM is rewritten as $\hat{\mathbf{R}}=\frac{1}{K} \mathbf{X} \mathbf{X}^{H}$. The matrix $\mathbf{X}$ is a function of the theoretical covariance matrix:

$$
\mathbf{X}=\mathbf{R}^{1 / 2} \mathbf{Y}=\left(\mathbf{I}_{m}+\mathbf{C}\right)^{1 / 2} \mathbf{Y}
$$

where $\mathbf{R}^{1 / 2}$ is the $m \times m$ Hermitian positive definite square root of the covariance matrix and the matrix $\mathbf{Y} \in \mathbb{C}^{m \times K}$ has iid entries $y_{i j} \sim \mathcal{C N}(0,1)$. Under the previous assumptions, the following properties are satisfied for this matrix: the probability law of $\mathbf{Y}$ is invariant by left multiplication with a deterministic unitary matrix. The eigenvalues empirical PDF of $\frac{1}{K} \mathbf{Y} \mathbf{Y}^{H}$ a.s. converges to the Marčenko-Pastur distribution [35] with support $\left[(1-\sqrt{c})^{2},(1+\sqrt{c})^{2}\right]$ and the maximum (resp. minimum) eigenvalue of $\frac{1}{K} \mathbf{Y} \mathbf{Y}^{H}$ a.s. tends to $(1+\sqrt{c})^{2}$ (resp. to $\left.(1-\sqrt{c})^{2}\right)$. From this last result, the convergence of the eigenvalues of the SCM are addressed through the convergence of the Stieltjes transform of the eigenvalues CDF and are given for the G-MUSIC model in [36].

Finally, a final assumption for the G-MUSIC model is needed to obtain the convergences of quadratic forms that are functions of estimated projectors. This will provide the value of $c$ which leads to separated clusters in $f(x)$ for all the theoretical eigenvalues of $\mathbf{R}$. This will be referred as the separation condition.

(As4) The ratio $c=m / K$ is chosen such that $1 / c$ is strictly greater than $\left\{\psi\left(\bar{f}_{i}\right): i \in \llbracket 1, t \rrbracket\right\}$ which depends on $m$, where $\psi(f)$ is defined in [22].

\footnotetext{
${ }^{2}$ Although the paper only considers Gaussian random variables, the results are still valid with random variables which have finite eighth order moments.
} 
Finally, the useful convergences of simple QFs (as $\left.\mathbf{s}_{1}^{H} \hat{\mathbf{\Pi}} \mathbf{s}_{2}\right)$ will be given in the next subsection.

2) Convergence of simple QFs: Under (As1-As4) and when $m, K \rightarrow \infty$ at the same rate $\left(m / K \rightarrow c \in \mathbb{R}^{+*}\right)$, then [22]:

$$
\boldsymbol{s}_{1}^{H} \hat{\boldsymbol{\Pi}}_{\mathrm{c}}^{\perp} \boldsymbol{s}_{2} \underset{\substack{m, K \rightarrow \infty \\ m / K \rightarrow c<\infty}}{\stackrel{\text { a.s. }}{\longrightarrow}} \boldsymbol{s}_{1}^{H} \overline{\boldsymbol{\Pi}}_{\mathrm{c}}^{\perp} \boldsymbol{s}_{2}
$$

with, $\overline{\boldsymbol{\Pi}}_{\mathrm{c}}^{\perp}=\sum_{i=1}^{m} \psi_{i} \boldsymbol{u}_{i} \boldsymbol{u}_{i}^{H}$ [22], and:

$$
\psi_{i}= \begin{cases}1-\frac{1}{m-r-1} \sum_{n=1}^{r}\left(\frac{1}{\lambda_{n}-1}-\frac{\mu_{m}}{\lambda_{n}-\mu_{m}}\right), & \text { if } i>r \\ \frac{1}{\lambda_{i}-1}-\frac{\mu_{m}}{\lambda_{i}-\mu_{m}}, & \text { else }\end{cases}
$$

where $\mu_{1} \geqslant \cdots \geqslant \mu_{m}$ are the eigenvalues of $\operatorname{diag}(\boldsymbol{\lambda})-$ $\frac{1}{K} \sqrt{\boldsymbol{\lambda}} \sqrt{\boldsymbol{\lambda}}^{T}$ and $\boldsymbol{\lambda}=\left[\lambda_{1}, \cdots, \lambda_{m}\right]^{T}$.

In the following, we denote:

$$
\overline{\mathbf{\Pi}}_{\mathrm{c}}^{\perp}=\mathbf{U} \Psi \mathbf{U}^{H},
$$

where $\mathbf{U}=\left[\mathbf{u}_{1} \ldots \mathbf{u}_{m}\right]$ and $\boldsymbol{\Psi}=\operatorname{diag}\left(\psi_{i}\right)_{i \in[1, m]}$

\section{Approximate Pfa And Pd of the LR-ANMF}

In this section, we derive an approximate Pfa and Pd for the LR-ANMF. The derivation is decomposed in these steps:

- first, we compute the convergence of the LR-ANMF in the large dimension regime in order to obtain an equivalent detector without a random projector. After some algebraic manipulations, we will obtain a ratio of two QFs which are function of real Gaussian random variables;

- from this ratio, we will compute an approximate distribution and we will present the algorithm to determine the parameters of this new distribution;

- finally the Pfa and the Pd will be evaluated from this approximate distribution.

\section{A. Approximate distribution of the LR-ANMF}

Let $\mathbf{x}$ be the signal under test. From Eq. (1), $\mathbf{x} \sim$ $\mathcal{C N}\left(\boldsymbol{\mu}_{x}, \mathbf{R}\right)$ where $\boldsymbol{\mu}_{x}=\mathbf{0}$ under $\mathcal{H}_{0}$ and $\boldsymbol{\mu}_{x}=\alpha \mathbf{d}$ under $\mathcal{H}_{1}$.

Proposition III.1 Equivalent detector LR-ANMF $\hat{\Lambda}_{L R}$ in a large dimension regime

Under (As1-As4) and when $m, K \rightarrow \infty$ at the same rate $\left(m / K \rightarrow c \in \mathbb{R}^{+*}\right)$, we have:

$$
\hat{\Lambda}_{\mathrm{LR}} \underset{\substack{m, K \rightarrow \infty \\ m / K \rightarrow c<\infty}}{\stackrel{\text { a.s. }}{\rightarrow}} \bar{\Lambda}=\frac{\tilde{\mathbf{z}}^{T} \tilde{\mathbf{A}} \tilde{\mathbf{z}}}{\tilde{\mathbf{z}}^{T} \tilde{\mathbf{H}} \tilde{\mathbf{z}}},
$$

with $\tilde{\mathbf{z}} \in \mathbb{R}^{2 m \times 1} \quad \in \quad$ and $\tilde{\mathbf{z}} \sim$ $\mathcal{N}\left(\boldsymbol{\mu}_{\tilde{\mathbf{z}}}=\sqrt{2}\left[\Re\left(\boldsymbol{\Lambda}^{-1 / 2} \mathbf{U}^{H} \boldsymbol{\mu}_{\mathbf{x}}\right)^{T}, \Im\left(\boldsymbol{\Lambda}^{-1 / 2} \mathbf{U}^{H} \boldsymbol{\mu}_{\mathbf{x}}\right)^{T}\right]^{T}, \mathbf{I}_{2 m}\right)$, $\boldsymbol{\Lambda}=\operatorname{diag}\left(\left[\lambda_{1}, \cdots, \lambda_{m}\right]\right)$ and

$$
\left\{\begin{array}{l}
\tilde{\mathbf{A}}=\left[\begin{array}{cc}
\Re(\mathbf{A}) & -\Im(\mathbf{A}) \\
\Im(\mathbf{A}) & \Re(\mathbf{A})
\end{array}\right] \in \mathbb{R}^{2 m \times 2 m} \\
\tilde{\mathbf{H}}=\left[\begin{array}{cc}
\mathbf{H} & \mathbb{D}_{m} \\
\mathbb{D}_{m} & \mathbf{H}
\end{array}\right] \in \mathbb{R}^{2 m \times 2 m}
\end{array}\right.
$$

where $\mathbf{A}=\mathbf{H}^{1 / 2} \mathrm{ff}^{H} \mathbf{H}^{1 / 2}, \mathbf{H}=\operatorname{diag}\left(\left[\eta_{1}, \cdots, \eta_{m}\right]\right), \eta_{i}=$ $\psi_{i} \lambda_{i}$ and $\mathbf{f}=\Psi^{1 / 2} \mathbf{U}^{H} \mathbf{d} /\left\|\Psi^{1 / 2} \mathbf{U}^{H} \mathbf{d}\right\|_{2}$.

\section{Proof: See appendix VI-A.•}

The expression $\bar{\Lambda}$ is now a ratio of two simple QFs, which are functions of real Gaussian random variables. The approximation by polynomial adjustment given in [31] is then fully adapted to this new form. The distribution of $\bar{\Lambda}$ under hypothesis $\mathcal{H}_{k}, k \in\{0,1\}$ and its approximation will be respectively denoted $f_{\bar{\Lambda}}\left(x \mid \mathcal{H}_{k}\right)$ and $\tilde{f}_{\bar{\Lambda}}\left(x \mid \mathcal{H}_{k}\right)$. Next, we have the following proposition:

Proposition III.2 Approximate distribution of $\hat{\Lambda}_{L R}$ in a large dimension regime

$f_{\bar{\Lambda}}\left(x \mid \mathcal{H}_{k}\right)$ is approximated by the following distribution:

$$
f_{\bar{\Lambda}}\left(x \mid \mathcal{H}_{k}\right) \approx \tilde{f}_{\bar{\Lambda}}\left(x \mid \mathcal{H}_{k}\right)=\varphi\left(x \mid \mathcal{H}_{k}\right) \sum_{i=0}^{d} \nu_{i \mid \mathcal{H}_{k}} x^{i},
$$

where $\varphi\left(x \mid \mathcal{H}_{k}\right)$ is a basic distribution (for example the exponential or the Beta distribution) of same support than $f_{\bar{\Lambda}}\left(x \mid \mathcal{H}_{k}\right)$ and $\tilde{f}_{\bar{\Lambda}}\left(x \mid \mathcal{H}_{k}\right), \nu_{i \mid \mathcal{H}_{k}}$ are the coefficients of the polynomial of adjustment and d its degree.

Proof: The proof is straightforward by following the result of [31].

\section{B. Evaluation of $\tilde{f}_{\bar{\Lambda}}\left(x \mid \mathcal{H}_{k}\right)$}

The choice of $d$ is important and is linked to the quality of the approximation but also to the computational cost (especially for $\left.\mathcal{H}_{1}\right)$. Next, the evaluation of $\tilde{f}_{\bar{\Lambda}}\left(x \mid \mathcal{H}_{k}\right)$ is performed in several steps:

- computation of the moments of $\bar{\Lambda}$;

- computation of the moments of $\varphi\left(x \mid \mathcal{H}_{k}\right)$;

- equalization of the moments of $\bar{\Lambda}$ and $\varphi\left(x \mid \mathcal{H}_{k}\right)$ in order to compute the elements $\left\{\nu_{i} \mid \mathcal{H}_{k}\right\}_{(1, d)}$.

It is important to notice that the support of $\varphi\left(x \mid \mathcal{H}_{k}\right)$ has to match with that of $f_{\bar{\Lambda}}\left(x \mid \mathcal{H}_{k}\right)$ and $\tilde{f}_{\bar{\Lambda}}\left(x \mid \mathcal{H}_{k}\right)$.

\section{Computation of the moments of $\bar{\Lambda}$ :}

In this paragraph, we compute the moments $\mu_{i \mid \mathcal{H}_{k}}^{(\bar{\Lambda})}$ of $\bar{\Lambda}$. They are determined by the following relation [31], [37] from the ratio of $\mathrm{Eq}(13)$ in the proposition III.1:

$$
\begin{aligned}
\mu_{i \mid \mathcal{H}_{k}}^{(\bar{\Lambda})}= & \frac{1}{\Gamma(i)} \exp \left(-\frac{\left\|\boldsymbol{\mu}_{\tilde{z}}\right\|_{2}^{2}}{2}\right) \int_{0}^{+\infty} y^{i-1} \frac{\partial^{i}}{\partial s^{i}}\left\{\left|\mathbf{I}_{2 m}-2 s \tilde{\mathbf{A}}+2 y \tilde{\mathbf{H}}\right|^{-1 / 2}\right. \\
& \left.\times \exp \left(\frac{1}{2} \boldsymbol{\mu}_{\tilde{\mathbf{z}}}^{T}\left(\mathbf{I}_{2 m}-2 s \tilde{\mathbf{A}}+2 y \tilde{\mathbf{H}}\right)^{-1} \boldsymbol{\mu}_{\tilde{\mathbf{z}}}\right)\right\}\left.\right|_{s=0} d y
\end{aligned}
$$

where $\Gamma($.$) is the Gamma function.$

Choice of the distribution of $\varphi\left(x \mid \mathcal{H}_{k}\right)$ and computation of its moments:

Since the detector $\hat{\Lambda}_{L R}$ takes values between $[0,1]$, it seems to be logical that its limit in the large dimension regime $\bar{\Lambda}$ takes the same values. In this case, the choice for $\varphi\left(x \mid \mathcal{H}_{k}\right)$ of a Beta distribution is interesting because its support is also $[0,1]$ and the expressions of its moments are simple. The non central moments linked to the Beta distribution are iteratively computed:

$$
\mu_{i \mid \mathcal{H}_{k}}^{(\varphi)}=\frac{\alpha_{k}+i-1}{\alpha_{k}+\beta_{k}+i-1} \mu_{i-1 \mid \mathcal{H}_{k}}^{(\varphi)},
$$


with $\mu_{1 \mid \mathcal{H}_{k}}^{(\varphi)}=\frac{\alpha_{k}}{\alpha_{k}+\beta_{k}}$ and where $\alpha_{k}$ and $\beta_{k}$ are the parameters of the Beta distribution:

$$
\begin{aligned}
& \alpha_{k}=-\mu_{1 \mid \mathcal{H}_{k}}^{(\bar{\Lambda})}+\frac{\left(1-\mu_{1 \mid \mathcal{H}_{k}}^{(\bar{\Lambda})}\right)\left(\mu_{1 \mid \mathcal{H}_{k}}^{(\bar{\Lambda})}\right)^{2}}{\mu_{2 \mid \mathcal{H}_{k}}^{\left(\bar{\Lambda}-\left(\mu_{1 \mid \mathcal{H}_{k}}^{(\bar{\Lambda}}\right)^{2}\right.}} \\
& \beta_{k}=-1-\alpha_{k}+\frac{\left(1-\mu_{1 \mid \mathcal{H}_{k}}^{(\bar{\Lambda})}\right) \mu_{1 \mid \mathcal{H}_{k}}^{(\bar{\Lambda})}}{\mu_{2 \mid \mathcal{H}_{k}}^{(\bar{\Lambda})}-\left(\mu_{1 \mid \mathcal{H}_{k}}^{(\bar{\Lambda})}\right)^{2}}
\end{aligned}
$$

Equalization of the moments of $\bar{\Lambda}$ and $\varphi\left(x \mid \mathcal{H}_{k}\right)$ :

This last step allows to compute the elements $\left\{\nu_{i} \mid \mathcal{H}_{k}\right\}_{\{0, d\}}$. For this computation, the non central moments of $\bar{\Lambda}$ are used to obtain those of $\tilde{f}_{\bar{\Lambda}}\left(x \mid \mathcal{H}_{k}\right)$ from the following relation [31]:

$$
\mu_{h \mid \mathcal{H}_{k}}^{(\bar{\Lambda})}=\sum_{i=0}^{d} \nu_{i \mid \mathcal{H}_{k}} \mu_{h+i \mid \mathcal{H}_{k}}^{(\varphi)}, \quad \forall h \in \llbracket 0, d \rrbracket,
$$

where $\mu_{h \mid \mathcal{H}_{k}}^{(\bar{\Lambda})}$ (resp. $\left.\mu_{h \mid \mathcal{H}_{k}}^{(\varphi)}\right), h \in \llbracket 1, d \rrbracket$ are the non central moments of order $h$ of $\bar{\Lambda}$ (resp. associated to $\varphi\left(x \mid \mathcal{H}_{k}\right)$ ) and $\mu_{0 \mid \mathcal{H}_{k}}^{(\bar{\Lambda})}=\mu_{0 \mid \mathcal{H}_{k}}^{(\varphi)}=1$. By setting $\nu_{k}=\left[\nu_{0 \mid \mathcal{H}_{k}} \nu_{1 \mid \mathcal{H}_{k}} \ldots \nu_{d \mid \mathcal{H}_{k}}\right]^{T}$, $\boldsymbol{\mu}_{k}^{(\bar{\Lambda})}=\left[\mu_{0 \mid \mathcal{H}_{k}}^{(\Lambda)} \mu_{1 \mid \mathcal{H}_{k}}^{(\bar{\Lambda})} \ldots \mu_{d \mid \mathcal{H}_{k}}^{(\bar{\Lambda})}\right]^{T}$ and

$$
\mathbf{M}_{k}=\left[\begin{array}{cccc}
\mu_{0 \mid \mathcal{H}_{k}}^{(\varphi)} & \mu_{1 \mid \mathcal{H}_{k}}^{(\varphi)} & \cdots & \mu_{d \mid \mathcal{H}_{k}}^{(\varphi)} \\
\mu_{1 \mid \mathcal{H}_{k}}^{(\varphi)} & \mu_{2 \mid \mathcal{H}_{k}}^{(\varphi)} & \cdots & \mu_{d+1 \mid \mathcal{H}_{k}}^{(\varphi)} \\
\vdots & \vdots & & \vdots \\
\mu_{d \mid \mathcal{H}_{k}}^{(\varphi)} & \mu_{d+1 \mid \mathcal{H}_{k}}^{(\varphi)} & \cdots & \mu_{2 d \mid \mathcal{H}_{k}}^{(\varphi)}
\end{array}\right]
$$

we have

$$
\boldsymbol{\nu}_{k}=\mathbf{M}_{k}^{-1} \boldsymbol{\mu}_{k}^{(\bar{\Lambda})} .
$$

We give the summary of the different steps in the following algorithm.

\section{Summary of the computation of the approximate distribution}

- Choice of initial parameters: $d$.

1. Preliminary calculus: $\mathbf{f}, \tilde{\mathbf{A}}$ and $\tilde{\mathbf{H}}$ according to Proposition III.1.

2. Computation of the $d$ non central moments $\mu_{i \mid \mathcal{H}_{k}}^{(\bar{\Lambda})}$ of $\bar{\Lambda}$ thanks to Eq (16).

3. Computation of the parameters of the base distribution, $\alpha_{k}$ and $\beta_{k}$ from Eq (18).

4. Computation of the $2 d$ non central moments $\mu_{i \mid \mathcal{H}_{k}}^{(\varphi)}$ from Eqs. (17).

5. Computation of the vector of $d+1$ coefficients $\boldsymbol{\nu}_{k}$ from $\mathrm{Eq}(20)$.

- Computation of the approximate density of probability $\tilde{f}_{\bar{\Lambda}}\left(x \mid \mathcal{H}_{k}\right)$, its cumulative distribution function, etc.

\section{Approximate Pfa and Pd of the LR-ANMF}

We now characterize the performance of the LR-ANMF detector by deriving the relations Pfa-threshold and $\mathrm{Pd}$ threshold. This derivation is based on the approximate distribution $\tilde{f}_{\bar{\Lambda}}\left(x \mid \mathcal{H}_{1}\right)$ computed under $\mathcal{H}_{0}$ and $\mathcal{H}_{1}$. These relations are given in the following proposition.
Proposition III.3 Approximate relations Pfa-threshold and Pd-threshold for the LR-ANMF detector

For a threshold denoted $\bar{\eta} \in[0,1]$ we have the following theoretical relations Pfa-threshold and Pd-threshold:

$$
\begin{gathered}
P f a \simeq \sum_{i=0}^{d} \nu_{i \mid \mathcal{H}_{0}} \frac{\Gamma\left(\alpha_{0}+\beta_{0}\right) \Gamma\left(i+\alpha_{0}\right)}{\Gamma\left(\alpha_{0}\right) \Gamma\left(i+\alpha_{0}+\beta_{0}\right)}\left(1-I_{\bar{\eta}}\left(i+\alpha_{0}, \beta_{0}\right)\right) \\
P d \simeq \sum_{i=0}^{d} \nu_{i \mid \mathcal{H}_{1}} \frac{\Gamma\left(\alpha_{1}+\beta_{1}\right) \Gamma\left(i+\alpha_{1}\right)}{\Gamma\left(\alpha_{1}\right) \Gamma\left(i+\alpha_{1}+\beta_{1}\right)}\left(1-I_{\bar{\eta}}\left(i+\alpha_{1}, \beta_{1}\right)\right),
\end{gathered}
$$

where $\alpha_{k}$ and $\beta_{k}$ are obtained from Eq (18).

Proof: See appendix VI-B. •

Finally, we have the approximate relations Pfa-threshold and Pd-threshold of the LR-ANMF as a function of the radar parameters.

\section{VALIDATIONS}

In this section, we validate the different propositions given in this paper. Since these results of theoretical performance are obtained whatever the tested target, we choose a steering vector $\mathbf{d}$ close to the clutter subspace. Therefore, we do not present the results of [19] to obtain the clearest figures because they are not valid in this configuration.

\section{A. Application presentation and parameters}

Since the disturbance has to be composed of a LR subspace plus a WGN, the jamming application is chosen. Indeed, the main purpose of this application is to detect a target with an uniform linear antenna composed of $m=20$ sensors despite the presence of $r$ jammers. Their response $c$ is composed of $r=3$ synthetic targets, similar to $\mathbf{d}(\theta)$, of Angles of Arrival (AoA) $\theta_{\mathbf{c}}$ equal to $-20^{\circ}, 0^{\circ}$ and $20^{\circ}$. Therefore, the rank $r$ of the covariance matrix of the low rank contribution, $\mathbf{C}$, is equal to 3 and the eigenvalues are chosen equal to $\gamma_{1}=6$, $\gamma_{2}=2$ and $\gamma_{3}=1$. The covariance matrix of the total noise is then defined as:

$$
\mathbf{R}=\frac{\alpha_{J N R}}{\operatorname{tr}(\boldsymbol{\Gamma})} \mathbf{C}+\gamma \mathbf{I}_{m},
$$

with $\boldsymbol{\Gamma}=\operatorname{diag}\left(\left[\gamma_{1}, \gamma_{2}, \gamma_{3}\right]\right), \gamma=1$ and where $\alpha_{J N R}$ is used to set the Jamming to Noise Ratio (JNR). The JNR and the SNR (signal $\mathbf{d}$ to noise ratio) are fixed to $10 \mathrm{~dB}$ and $18 \mathrm{~dB}$ respectively, $K=r+1$ which results in $c_{m}=5$ and the AoA of the data $\mathbf{x}$ under test is $\theta_{\mathbf{d}}=20.1^{\circ}$. We notice that the tested data is very close to the jammer subspace. The wavelength is fixed at $0.667 \mathrm{~m}$.

\section{B. Validation of the approximate distribution of the LR-ANMF}

In this subsection we validate the asymptotic equivalence of the LR-ANMF test given in the propositions III.1 and III.2. The goal is first to measure the impact of the asymptotic approximation in the large dimension regime on the distribution and next to validate the approximation by polynomial adjustment in particular with a small value of $d$.

We visualize in Fig. 1 the ECDF (Empirical Cumulative Distribution Function) of $\bar{\Lambda}$ of Eq. (13) and the CDF of the approximate distribution, $\tilde{f}_{\bar{\Lambda}}$ of Eq. (15), with a minimal 
approximation degree $d=2$. We also compare them to the ECDFs of the LR-ANMF, $\hat{\Lambda}_{L R}$ of Eq. (6), and the LR-NMF detector (built with the theoretical projector and denoted $\Lambda_{L R}$ ). All these quantities are first evaluated under the $\mathcal{H}_{0}$ hypothesis in Fig. 1(a) and under the $\mathcal{H}_{1}$ hypothesis in Fig. 1(b). To quantify the distance between two ECDFs or one ECDF and one CDF, we use the Cramer-Von Mises distance defined by:

$$
d_{c v m}=E_{x}\left[\left|F_{1}(x)-F_{2}(x)\right|^{2}\right],
$$

where the expectation is taken over $x$ and $F_{1}(x)$ and $F_{2}(x)$ are two ECDF (or CDF). In the following, the Cramer-Von Mises distances will be evaluated with $10^{5}$ trials.

Under $\mathcal{H}_{0}, d_{\text {cvm }}$ between the ecdfs of the LR-ANMF $\hat{\Lambda}_{L R}$ and that of the LR-NMF $\Lambda_{L R}$ is equal to 0.06 and $d_{c v m}$ between the ecdfs of the LR-ANMF $\hat{\Lambda}_{L R}$ and that of $\bar{\Lambda}$ is equal to 0.007: we first notice that the approximation in a large dimension regime provides an ECDF much closer to the LRANMF ecdf than the LR-NMF one. Moreover, $d_{c v m}$ between the ecdf of $\hat{\Lambda}_{L R}$ and the cdf derived from the distribution $\tilde{f}_{\bar{\Lambda}}$ is equal to around $4.10^{-5}$ : we conclude that even for a small degree of approximation, the theoretical approximation proposed in this paper is very accurate.

Under $\mathcal{H}_{1}, d_{\text {cvm }}$ between the ecdfs of the LR-ANMF $\hat{\Lambda}_{L R}$ and that of the LR-NMF $\Lambda_{L R}$ is equal to 0.224 and $d_{c v m}$ between the ecdfs of the LR-ANMF $\hat{\Lambda}_{L R}$ and that of $\bar{\Lambda}$ is equal to 0.002 : as for $\mathcal{H}_{0}$, the approximation in a large dimension regime provides an ECDF closer to the LR-ANMF ecdf than the LR-NMF one. Under $\mathcal{H}_{1}, d_{\text {cvm }}$ between the ecdf of $\hat{\Lambda}_{L R}$ and the cdf derived from the distribution $\tilde{f}_{\bar{\Lambda}}$ is approximately $3.7 .10^{-4}$ : the approximation $\tilde{f}_{\bar{\Lambda}}$ is less accurate than under $\mathcal{H}_{0}$ but the result allows to conclude that the theoretical result proposed in this paper provides a good approximation of the behavior of $\bar{\Lambda}$.

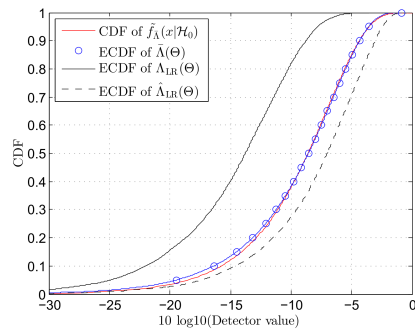

(a) Under $\mathcal{H}_{0}$

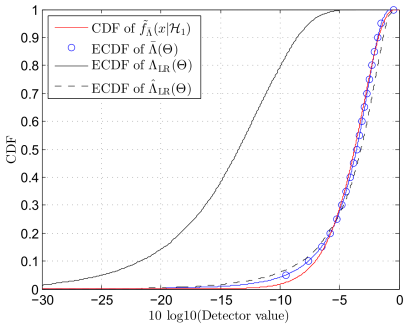

(b) Under $\mathcal{H}_{1}$
Fig. 1. CDF of the approximate distribution $\tilde{f}_{\bar{\Lambda}}$ with $d=2$, ECDF of $\bar{\Lambda}$, of the LR-ANMF $\hat{\Lambda}_{L R}$ and of the LR-NMF $\Lambda_{L R} \cdot m=20, K=r+1$ and $\theta=20,1^{\circ}$. The JNR is fixed at $10 \mathrm{~dB} .10^{5}$ trials have been used to evaluate the ECDFs.

\section{Validation of the approximate Pfa and Pd of the LR-ANMF}

We are here interested in the validation of the Pfathreshold and Pd-threshold relations given in proposition III.3.

First, Fig.2(a) compares the Pfa-threshold relations of the LR-ANMF, of the LR-NMF and of Eq. (21) derived from the distribution $\tilde{f}_{\bar{\Lambda}}$. Similarly to the previous subsection, we conclude that the Pfa-threshold relation (21) is a correct approximation in particular for small Pfa. Next, Fig.2(b) shows the Pd-threshold relations of the LR-ANMF, of the LR-NMF and of Eq. (22) derived from the distribution $\tilde{f}_{\bar{\Lambda}}$. Like for the Pfa-threshold, we notice that the relation (22) is a good approximation of the Pd of the LR-ANMF.

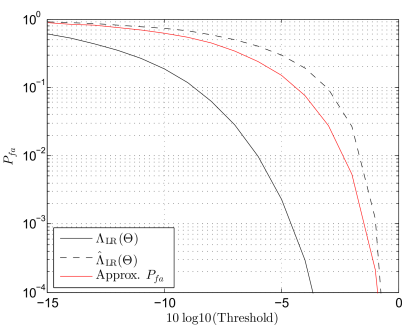

(a) Pfa-threshold relations

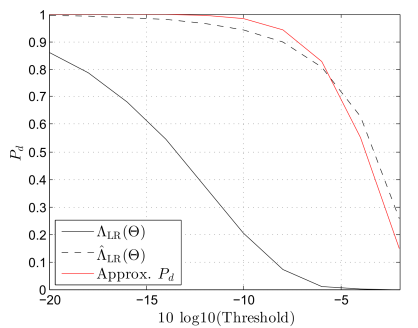

(b) Pd-threshold relations
Fig. 2. Pfa-threshold and Pd-threshold relations of LR-NMF $\Lambda_{L R}$, of LRANMF $\hat{\Lambda}_{L R}$ and of Eq. (22) derived from the distribution $\tilde{f}_{\bar{\Lambda}} \cdot m=20$, $K=r+1$ and $\theta=20,1^{\circ}$. For the LR-NMF and LR-ANMF, $10^{5}$ trials have been used to evaluated the Pfa and the Pd. The JNR and the SNR are fixed at $10 \mathrm{~dB}$ and $18 \mathrm{~dB}$.

In Fig. 3, we show the Pd as a function of the SNR for a fixed Pfa $=10^{-2}$. We notice that the LR-NMF is not able to predict the performance of the LR-ANMF. Even if we have an error between the theoretical result and the Monte Carlo simulations, we conclude that it provides a correct approximation of the detection performance when the target under test is near the LR subspace.

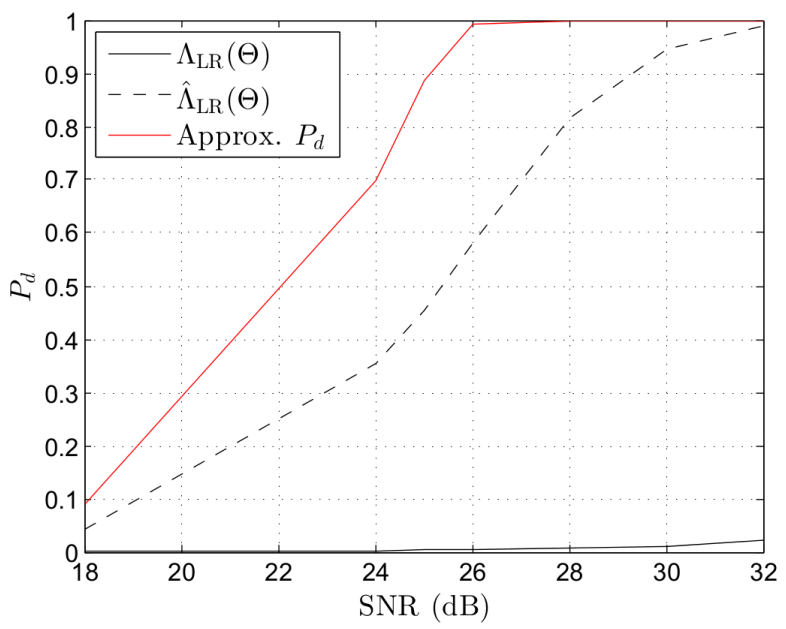

Fig. 3. Pd-SNR relations of LR-NMF ${\underset{\sim}{\Lambda_{L R}}}_{L}$, of LR-ANMF $\hat{\Lambda}_{L R}$ and of Eq. (22) derived from the distribution $\tilde{f}_{\bar{\Lambda}} \cdot m=20, K=r+1$ and $\theta=20,1^{\circ}$. For the LR-NMF and LR-ANMF, $10^{5}$ trials have been used to evaluated the Pd. The JNR is fixed at $10 \mathrm{~dB}$ and the Pfa is fixed at $10^{-3}$.

Finally, the table I presents the average calculation time for one point of the Pfa-threshold relation with $10^{4}$ iterations when the moments are already calculated ${ }^{3}$. We notice that the approximation (21) given in this paper allows to strongly reduce the computational cost compared to a classic Monte Carlo simulation. But, this equation requires computing the moment generator function of Eq. (16). If this step has a computational cost of one hour under $\mathcal{H}_{0}$, it becomes prohibitive under $\mathcal{H}_{1}$

\footnotetext{
${ }^{3}$ The computer used has an Intel(R) Core(TM) i7-3667U CPU @ 2,00GHz processor and RAM memory of $8 \mathrm{Go}$.
} 


\begin{tabular}{|l|c|c|}
\hline & $K=r+1$ & $K=2 r$ \\
\hline With $\hat{\Lambda}_{\mathrm{LR}}$ & $3,0342 \mathrm{~s}$ & $3,0188 \mathrm{~s}$ \\
\hline With the theoretical relation (Eq.(21)) & $0,0252 \mathrm{~s}$ & $0,0240 \mathrm{~s}$ \\
\hline
\end{tabular}

TABLE I

AVERAGE CALCUlation TIME FOR ONE POINT OF THE PFA-THRESHOLD RELATION WITH $10^{4}$ ITERATIONS AND WHEN THE PFA IS SET AT $10^{-4}$.

(around 2 days). Therefore, the approximation (21) allows to obtain a threshold for a given Pfa with a small computation time. On the other hand, Monte Carlo trials are more suitable to evaluate the $\mathrm{Pd}$.

\section{CONCLUSION}

In this paper, we derived the approximate Pfa-threshold and Pd-threshold relations of the LR-ANMF in a large dimension regime. Compared to the previous work [19], the tested target is assumed to be close to the LR-subspace. In this configuration, we showed that the LR-ANMF lost its CFAR property and its performance depended on the distance between the target and the LR subspace. More generally, this paper illustrated that the tools of RMT are useful for deriving an approximation of a complex statistic form like the LR-ANMF under conditions where the classical tools of asymptotic analysis are unable to provide an interesting result.

We validated this theoretical result in a jamming application where the data under test is close to the LR subspace. Finally, we illustrated that the computational cost is low under $\mathcal{H}_{0}$, which allows a threshold to be easily obtained for a chosen Pfa without the use of Monte Carlo simulations. Unfortunately the computational cost under $\mathcal{H}_{1}$ is too high for a practical use.

\section{ACKNOWLEDGMENT}

We thank the ONERA agency and SONDRA Lab to partially fund this work.

\section{APPENDIX}

\section{A. Proof of proposition III.1}

From the convergence results for the QFs given in subsection II-C2 (under hypotheses (AS1-AS4)) and the continuous mapping theorem we directly have:

$$
\hat{\Lambda}_{\mathrm{LR}} \underset{\substack{m, K \rightarrow \infty \\ m / K \rightarrow c<\infty}}{\stackrel{\text { p.s. }}{\longrightarrow}} \bar{\Lambda}=\frac{\left|\boldsymbol{d}^{H} \overline{\boldsymbol{\Pi}}_{\mathrm{c}}^{\perp} \boldsymbol{x}\right|^{2}}{\left(\boldsymbol{d}^{H} \overline{\boldsymbol{\Pi}}_{\mathrm{C}}^{\perp} \boldsymbol{d}\right)\left(\boldsymbol{x}^{H} \overline{\boldsymbol{\Pi}}_{\mathrm{C}}^{\perp} \boldsymbol{x}\right)} .
$$

We notice that the LR-ANMF test is not consistent in a large dimension regime.

Next, we transform the previous equation in a ratio of two QFs, which are functions of complex Gaussian random variables:

$$
\bar{\Lambda}=\frac{\left|\boldsymbol{d}^{H} \mathbf{U} \boldsymbol{\Psi} \mathbf{U}^{H} \boldsymbol{x}\right|^{2}}{\left(\boldsymbol{d}^{H} \mathbf{U} \boldsymbol{\Psi} \mathbf{U}^{H} \boldsymbol{d}\right)\left(\boldsymbol{x}^{H} \mathbf{U} \boldsymbol{\Psi} \mathbf{U}^{H} \boldsymbol{x}\right)}=\frac{\left|\mathbf{e}^{H} \mathbf{y}\right|^{2}}{\left(\mathbf{e}^{H} \mathbf{e}\right)\left(\mathbf{y}^{H} \mathbf{y}\right)},
$$

with $\mathbf{e}=\boldsymbol{\Psi}^{1 / 2} \mathbf{U}^{H} \mathbf{d}, \mathbf{y}=\boldsymbol{\Psi}^{1 / 2} \mathbf{U}^{H} \mathbf{x} \sim \mathcal{C N}\left(\boldsymbol{\mu}_{\mathbf{y}}=\right.$ $\left.\boldsymbol{\Psi}^{1 / 2} \mathbf{U}^{H} \boldsymbol{\mu}_{\mathbf{x}}, \mathbf{H}\right), \mathbf{H}=\boldsymbol{\Lambda} \boldsymbol{\Psi}$ and $\boldsymbol{\Lambda}=\operatorname{diag}\left(\left[\lambda_{1}, \cdots, \lambda_{m}\right]\right)$.
Therefore,

$$
\begin{aligned}
\bar{\Lambda} & =\frac{\left|\mathbf{e}^{H} \mathbf{H}^{1 / 2} \mathbf{z}\right|^{2}}{\left(\mathbf{e}^{H} \mathbf{e}\right)\left(\mathbf{z}^{H} \mathbf{H z}\right)} \\
& =\frac{\mathbf{z}^{H} \mathbf{H}^{1 / 2} \mathbf{e e}^{H} \mathbf{H}^{1 / 2} \mathbf{z}}{\left(\mathbf{e}^{H} \mathbf{e}\right)\left(\mathbf{z}^{H} \mathbf{H z}\right)} \\
& =\frac{\mathbf{z}^{H} \mathbf{H}^{1 / 2} \mathbf{f f}^{H} \mathbf{H}^{1 / 2} \mathbf{z}}{\mathbf{z}^{H} \mathbf{H z}} \\
& =\frac{\mathbf{z}^{H} \mathbf{A} \mathbf{z}}{\mathbf{z}^{H} \mathbf{H z}},
\end{aligned}
$$

with $\mathbf{z}=\mathbf{H}^{-1 / 2} \mathbf{y} \sim \mathcal{C N}\left(\boldsymbol{\mu}_{\mathbf{z}}=\boldsymbol{\Lambda}^{-1 / 2} \mathbf{U}^{H} \boldsymbol{\mu}_{\mathbf{x}}, \mathbf{I}_{m}\right), \mathbf{f}=$ $\mathbf{e} /\|\mathbf{e}\|_{2}$ and $\mathbf{A}=\mathbf{H}^{1 / 2} \mathrm{ff}^{H} \mathbf{H}^{1 / 2}$.

Finally, it is easy to transform the previous relation which is in a complex form into an equivalent real form:

$$
\bar{\Lambda}=\frac{\mathbf{z}^{H} \mathbf{A} \mathbf{z}}{\mathbf{z}^{H} \mathbf{H z}}=\frac{\tilde{\mathbf{z}}^{T} \tilde{\mathbf{A}} \tilde{\mathbf{z}}}{\tilde{\mathbf{z}}^{T} \tilde{\mathbf{H}} \tilde{\mathbf{z}}}
$$

with

$$
\left\{\begin{array}{l}
\tilde{\mathbf{z}}=\sqrt{2}\left[\Re(\mathbf{z})^{T}, \Im(\mathbf{z})^{T}\right]^{T} \in \mathbb{R}^{2 m \times 1} \\
\tilde{\mathbf{A}}=\left[\begin{array}{ll}
\Re(\mathbf{A}) & -\Im(\mathbf{A}) \\
\Im(\mathbf{A}) & \Re(\mathbf{A})
\end{array}\right] \in \mathbb{R}^{2 m \times 2 m} \\
\tilde{\mathbf{H}}=\left[\begin{array}{cc}
\mathbf{H} & \mathbb{D}_{2 m} \\
\mathbb{D}_{2 m} & \mathbf{H}
\end{array}\right] \in \mathbb{R}^{2 m \times 2 m}
\end{array},\right.
$$

with $\tilde{\mathbf{z}} \sim \mathcal{N}\left(\boldsymbol{\mu}_{\tilde{\mathbf{z}}}=\sqrt{2}\left[\Re\left(\boldsymbol{\mu}_{\mathbf{z}}\right)^{T}, \Im\left(\boldsymbol{\mu}_{\mathbf{z}}\right)^{T}\right]^{T}, \mathbf{I}_{2 m}\right)$.

\section{B. Proof of proposition II.I}

We have by definition of the Pfa and the Pd:

$$
\begin{aligned}
P_{f a}, P_{d} & \simeq \int_{\bar{\eta}}^{+\infty} \tilde{f}_{\bar{\Lambda}}\left(x \mid \mathcal{H}_{k}\right) d x \\
& =\int_{\bar{\eta}}^{+\infty} \varphi\left(x \mid \mathcal{H}_{k}\right) \sum_{i=0}^{d} \nu_{i \mid \mathcal{H}_{k}} x^{i} d x \\
& =\sum_{i=0}^{d} \nu_{i \mid \mathcal{H}_{k}} P_{i}\left(\bar{\eta} \mid \mathcal{H}_{k}\right) d x
\end{aligned}
$$

with $P_{i}\left(\bar{\eta} \mid \mathcal{H}_{k}\right)=\int_{\bar{\eta}}^{+\infty} x^{i} \varphi\left(x \mid \mathcal{H}_{k}\right) d x$ and $k \in\{0,1\}$. Since $\varphi\left(x \mid \mathcal{H}_{k}\right)$ is a Beta distribution, then

$$
\begin{aligned}
P_{i}\left(\bar{\eta} \mid \mathcal{H}_{k}\right) & =\int_{\bar{\eta}}^{+\infty} x^{i} \frac{\Gamma\left(\alpha_{k}+\beta_{k}\right)}{\Gamma\left(\alpha_{k}\right) \Gamma\left(\beta_{k}\right)} x^{\alpha_{k}-1}(1-x)^{\beta_{k}-1} \mathbb{1}_{[0,1]} d x \\
& =\frac{\Gamma\left(\alpha_{k}+\beta_{k}\right)}{\Gamma\left(\alpha_{k}\right) \Gamma\left(\beta_{k}\right)} \int_{\bar{\eta}}^{+\infty} x^{i+\alpha_{k}-1}(1-x)^{\beta_{k}-1} \mathbb{1}_{[0,1]} d x \\
& =\frac{\Gamma\left(\alpha_{k}+\beta_{k}\right)}{\Gamma\left(\alpha_{k}\right) \Gamma\left(\beta_{k}\right)} \int_{\bar{\eta}}^{+\infty} \frac{\Gamma\left(i+\alpha_{k}\right) \Gamma\left(\beta_{k}\right)}{\Gamma\left(i+\alpha_{k}+\beta_{k}\right)} \mathcal{B}\left(i+\alpha_{k}, \beta_{k}\right) d x \\
& =\frac{\Gamma\left(\alpha_{k}+\beta_{k}\right) \Gamma\left(i+\alpha_{k}\right)}{\Gamma\left(\alpha_{k}\right) \Gamma\left(i+\alpha_{k}+\beta_{k}\right)} \int_{\bar{\eta}}^{+\infty} \mathcal{B}\left(i+\alpha_{k}, \beta_{k}\right) d x \\
& =\frac{\Gamma\left(\alpha_{k}+\beta_{k}\right) \Gamma\left(i+\alpha_{k}\right)}{\Gamma\left(\alpha_{k}\right) \Gamma\left(i+\alpha_{k}+\beta_{k}\right)}\left(1-\int_{-\infty}^{\bar{\eta}} \mathcal{B}\left(i+\alpha_{k}, \beta_{k}\right) d x\right) \\
& =\frac{\Gamma\left(\alpha_{k}+\beta_{k}\right) \Gamma\left(i+\alpha_{k}\right)}{\Gamma\left(\alpha_{k}\right) \Gamma\left(i+\alpha_{k}+\beta_{k}\right)}\left(1-I_{\bar{\eta}}\left(i+\alpha_{k}, \beta_{k}\right)\right)
\end{aligned}
$$

where $I_{\bar{\eta}}$ (resp. $\mathcal{B}$ ) is the regularized incomplete beta function (resp. the beta function). Therefore, the proof is finished by using the properties of $I_{\bar{\eta}}$.

\section{REFERENCES}

[1] E. Kelly, "An adaptive detection algorithm," IEEE Trans. on Aero. and Elec. Syst., vol. 22, no. 1, pp. 115-127, march 1986.

[2] F. Robey, D. Fuhrmann, E. Kelly, and R. Nitzberg, "A CFAR adaptive matched filter detector," IEEE Trans. on Aero. and Elec. Syst., vol. 28, no. 2, pp. $208-216,1992$.

[3] L. Scharf and B. Friedlander, "Matched subspace detectors," IEEE Trans. Signal Process., vol. 42, no. 8, pp. 2146-2157, august 1994. 
[4] S. Kraut, L. Scharf, and L. McWhorter, "Adaptive subspace detectors," IEEE Trans. Signal Process., vol. 49, no. 1, pp. 1-16, january 2001.

[5] I. Reed, J. Mallett, and L. Brennan, "Rapid convergence rate in adaptive arrays," IEEE Trans. on Aero. and Elec. Syst., vol. AES-10, no. 6, pp. 853 - 863, November 1974.

[6] L. B. Fertig, "Analytical expressions for space-time adaptive processing (STAP) performance," IEEE Trans. on Aero.and Elect. Syst., vol. 51, no. 1 , pp. $442-453$, January 2015.

[7] O. Ledoit and M. Wolf, "A well-conditioned estimator for largedimensional covariance matrices," Journal of multivariate analysis, vol. 88, pp. 365-411, 2004.

[8] L. Cai and H. Wang, "A persymmetric multiband GLR algorithm," IEEE Trans. on Aero. and Elec. Syst., vol. 30, no. 3, pp. 806-816, July 1992.

[9] G. Pailloux, P. Forster, J. Ovarlez, and F. Pascal, "Persymmetric adaptive radar detectors," IEEE Trans. on Aero. and Elec. Syst., vol. 47, no. 4 pp. 2376-2390, October 2011.

[10] G. Ginolhac, P. Forster, F. Pascal, and J. Ovarlez, "Exploiting persymmetry for low-rank space time adaptive processing," Signal Processing, vol. 97, no. 4, pp. 242 - 251, April 2014.

[11] A. D. Maio, D. Orlondo, C. Hao, and G. Foglia, "Adaptive detection of point-like targets in spectrally symmetric interference," IEEE Trans. Signal Process., vol. 64, no. 12, pp. 3207 - 3220, June 2016.

[12] C. Hao, D. Orlondo, , G. Foglia, and G. Giunta, "Knowledge-based adaptive detection: Joint exploitation of clutter and system symmetry properties," IEEE Signal Process. Letters, vol. 23, no. 10, pp. $1489-$ 1493, October 2016.

[13] M. Rangaswamy, F. Lin, and K. Gerlach, "Robust adaptive signal processing methods for heterogeneous radar clutter scenarios," Signal Processing, vol. 84, pp. 1653 - 1665, 2004.

[14] I. Kirsteins and D. Tufts, "Adaptive detection using a low rank approximation to a data matrix," IEEE Trans. on Aero. and Elec. Syst., vol. 30 pp. $55-67,1994$.

[15] A. Haimovich, "Asymptotic distribution of the conditional signal-tonoise ratio in an eigenanalysis-based adaptive array," IEEE Trans. on Aero. and Elec. Syst., vol. 33, pp. 988 - 997, 1997.

[16] A. Dogandzic and B. Zhang, "Complex signal amplitude estimation and adaptive detection in unknown low-rank interference," in Proceedings of Asilomar Conference, Pacific Grove, CA, USA, November 2006.

[17] _ "Bayesian complex amplitude estimation and adaptive matched filter detection in low-rank interference," IEEE Trans. Signal Process., vol. 55, no. 3, pp. 1176 - 1182, march 2007.

[18] J.-F. Degurse, L. Savy, and S. Marcos, "Reduced-rank STAP for target detection in heterogeneous environments," IEEE Trans. on Aero. and Elec. Syst., vol. 50, no. 2, pp. 1153 - 1162, april 2014.

[19] G. Ginolhac and P. Forster, "Approximate distribution of the lowrank adaptive normalized matched filter test statistic under the null hypothesis," IEEE Trans. on Aero. and Elect. Syst., vol. 64, no. 5, pp. -, November 2016

[20] C. Peckham, A. Haimovich, T. Ayoub, J. Goldstein, and I. Reed, "Reduced-rank STAP performance analysis," IEEE Trans. on Aero. and Elec. Syst., vol. 36, no. 2, pp. 664 - 676, April 2000.

[21] G. Ginolhac, P. Forster, F. Pascal, and J. Ovarlez, "Performance of two low-rank STAP filters in a heterogeneous noise," IEEE Trans. Signal Process., vol. 61, no. 1, pp. 57 - 61, January 2013.

[22] X. Mestre, "Improved estimation of eigenvalues and eigenvectors of covariance matrices using their sample estimates," IEEE Transactions on Information Theory, vol. 54, no. 11, pp. 5113 - 5129, November 2008.

[23] _ - "On the asymptotic behavior of the sample estimates of eigenvalues and eigenvectors of covariance matrices," IEEE Transactions on Signal Processing, vol. 56, no. 11, pp. 5353 - 5368, November 2008.

[24] P. Vallet, P. Loubaton, and X. Mestre, "Improved subspace estimation for multivariate observations of high dimension: the deterministic signal case," IEEE Trans. on Information Theory, vol. 58, no. 2, pp. $1043-$ 1068, Feb. 2012.

[25] R. Couillet and W. Hachem, "Fluctuations of spiked random matrix models and failure diagnosis in sensor networks," IEEE Transactions on Information Theory, vol. 59, no. 1, pp. 509 - 525, 2013.

[26] X. Mestre and M. Lagunas, "Modified subspace algorithms for DoA estimation with large arrays," IEEE Transactions on Information Theory, vol. 56, no. 2, pp. 598 - 614, February 2008.

[27] W. Hachem, P. Loubaton, X. Mestre, J. Najim, and P. Vallet, "A subspace estimator of fixed rank perturbations of large random matrices," Journal of Multivariate Analysis, vol. 114, pp. 427 - 447, 2013.

[28] R. Couillet, F. Pascal, and J. Silverstein, "Robust estimates of covariance matrices in the large dimensional regime," IEEE Transactions on Information Theory, vol. 60, no. 11, pp. 7269 - 7278, 2014, http://arxiv.org/abs/1204.5320.

[29] A. Combernoux, F. Pascal, G. Ginolhac, and M. Lesturgie, "Performances of low rank detectors based on random matrix theory with application to STAP," International Radar Conference, Lille, France, Oct. 2014.

[30] — "Asymptotic performance of the low rank adaptive normalized matched filter in a large dimensional regime," IEEE International Conference on Acoustics, Speech and Signal Processing (ICASSP), South Brisbane, QLD, Australia, Apr. 2015.

[31] A. Mohsenipour and S. Provost, "Approximating the distributions of singular quadratic expressions and their ratios," Journal of Iranian Statistical Society, vol. 11, no. 2, pp. 147 - 171, June 2012.

[32] L. E. Brennan and F. Staudaher, "Subclutter visibility demonstration," RL-TR-92-21, Adaptive Sensors Incorporated, Tech. Rep., 1992.

[33] N. Goodman and J. Stiles, "On clutter rank observed by arbitrary arrays," IEEE Transactions on Signal Processing, vol. 55, no. 1, pp. 178-186, Jan 2007.

[34] R. Nadakuditi and J. Silverstein, "Fundamental limit of sample generalized eigenvalue based detection of signals in noise using relatively few signal-bearing and noise-only samples," IEEE Journal of Selected Topics in Signal Processing, vol. 4, no. 3, pp. 468 - 480, 2010.

[35] V. Marčenko and L. Pastur, "Distributions of eigenvalues for some sets of random matrices," Mathematics of the USSR-Sbornik, vol. 1, no. 4, pp. 457 - 483, April 1967.

[36] J. Silverstein, "Strong convergence of the empirical distribution of eigenvalues of large dimensional random matrices," J. Multivar. Anal., vol. 55 , pp. $331-339,1995$.

[37] J. Magnus, "The exact moments of a ratio of quadratic forms in normal variables," Annales d'économie et de statistique, no. 4, pp. 95 - 109, 1986.

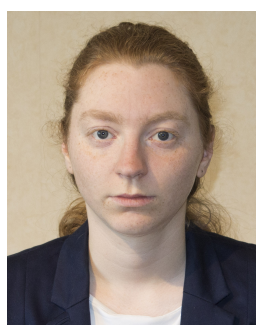

Alice Combernoux received the Master's degree ("Advanced Systems in Radiocommunications") and the degree of Electrical Engineer in Telecommunications from Supélec, Gif-sur-Yvette, France, in 2012. Then, she received the Ph.D. degree of Science Engineering (Signal and Image Processing speciality), from University Paris-Saclay in 2016. From April 2016 to April 2017, she was Engineer at ONERA, Palaiseau, France. From May 2017, she is a Research and Development Engineer at Thales DMS France, Brest, France. Her research interest include estimation, detection and classification with applications to array and image processing and radar/sonar. 


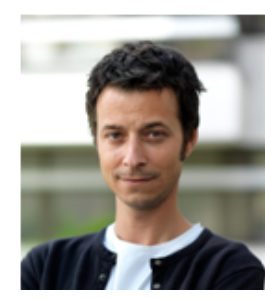

Frédéric Pascal (S'04, M'06, SM'14) received the Master's degree ("Probabilities, Statistics and Applications: Signal, Image et Networks") with merit, in Applied Statistics from University Paris VII Jussieu, Paris, France, in 2003. Then, he received the $\mathrm{Ph} . \mathrm{D}$. degree of Signal Processing, from University Paris X ? Nanterre in 2006. From November 2006 to February 2008, he made a post-doctoral position in the Signal Processing and Information team of the laboratory SATIE, CNRS, ENS Cachan, France. Between March 2008 and December 2011 (resp. Jan. 2012 ? Dec. 2013), he was an Assistant Professor (resp. Associate Professor) in SONDRA at CentraleSupélec. In 2012, he obtained a Research Directorship Habilitation (HDR) thesis in Signal Processing from the University of ParisSud. Between Aug. 2013 and Aug. 2014, he was a Visiting Associate Professor in the ECE department at the National University of Singapore. From Jan. 2014, Frederic Pascal is a full Professor in the L2S laboratory at CentraleSupélec. From Jan. 2017, he is the head of the ?Signals and Statistics? group of L2S. He is also the coordinator of the data science activities at CentraleSupélec and the chair holder of the Givaudan chair on data sciences. From Sept. 2017, he is in the Executive Committee of the DATAIA institute as the Program Coordinator. From 2015 to 2017, Frederic Pascal was the Chair of the EURASIP SAT in Theoretical and Methodological Trends in Signal Processing (TMTSP) and he is a member of the IEEE Signal Processing Society SAM technical committee (Jan. 2015-present). Frederic Pascal serves as an Associate Editor for the IEEE Transactions on Signal Processing (2015-2018), for the EURASIP Journal on Advances in Signal Processing (2015-present) and for Elsevier Signal Processing (2018-present). His research interests contain estimation, detection and classification for statistical signal processing and applications in radar and image processing. He is the author / coauthor of more than one hundred papers in the top journals and conferences in Signal Processing, Image Processing and Statistics.

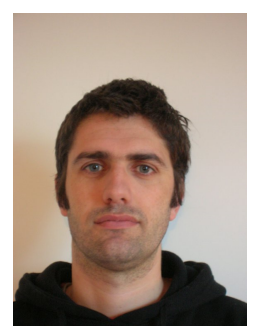

Guillame Ginolhac (S'98,M'01,SM'12) received the Master's degree of Electrical Engineer, in 1997 and the Ph.D. degree of Signal Processing, in 2001 both from the Grenoble-INP university (France). From 2002 to 2012, he was an associate professor in SATIE Lab and University Paris Nanterre. In 2011, he obtained a Research Directorship Habilitation (HDR) thesis in Signal Processing from the ENS Cachan institute. From 2012, Guillaume Ginolhac is a full professor in LISTIC Lab at University Savoie Mont-Blanc. From July 2018, he is the head of the "Connaissances, Images, Télédétection" group of LISTIC. His research interests include estimation and detection theory for statistical signal processing and applications to array processing and radar/sonar. 